\title{
TOWARDS EFFICIENCY OF OBLIQUE IMAGES ORIENTATION
}

\author{
W. Ostrowski ${ }^{\text {a*}}, \mathrm{K}$. Bakuła ${ }^{\text {a }}$ \\ ${ }^{a}$ Department of Photogrammetry, Remote Sensing and Spatial Information Systems, Faculty of Geodesy and Cartography, Warsaw \\ University of Technology, Poland - (w.ostrowski, k.bakula)@gik.pw.edu.pl
}

KEY WORDS: oblique, oblique images, orientation, adjustment, bundle, block

\begin{abstract}
:
Many papers on both theoretical aspects of bundle adjustment of oblique images and new operators for detecting tie points on oblique images have been written. However, only a few achievements presented in the literature were practically implemented in commercial software. In consequence often aerial triangulation is performed either for nadir images obtained simultaneously with oblique photos or bundle adjustment for separate images captured in different directions. The aim of this study was to investigate how the orientation of oblique images can be carried out effectively in commercial software based on the structure from motion technology. The main objective of the research was to evaluate the impact of the orientation strategy on both duration of the process and accuracy of photogrammetric 3D products. Two, very popular software: Pix4D and Agisoft Photoscan were tested and two approaches for image blocks were considered. The first approach based only on oblique images collected in four directions and the second approach included nadir images. In this study, blocks for three test areas were analysed. Oblique images were collected with medium-format cameras in maltan cross configuration with registration of GNSS and INS data. As a reference both check points and digital surface models from airborne laser scanning were used.
\end{abstract}

\section{INTRODUCTION}

Oblique airborne imagery become more and more popular photogrammetric datasets which is obviously related with rising market of their applications. The major advantage of oblique images from the consumer point of view is their 'natural' view which is much easier to interpret for non-expert users (Remondino and Gerke, 2015). These type of images have been successfully used for years in applications which do not demand high accuracy (Höhle, 2008). Because of that for many years enough accurate way of determining elements of exterior orientation parameters for oblique images was direct referencing. However, such a solution is insufficient for some applications (Grenzdorffer et at. 2008, Rupnik et al. 2015), and recently there has been a growing number of applications which need higher accuracies of measurement, like 3D City Modelling (Haala et al. 2015) and another verification or extraction information about urban environment (Rau et al. 2015, Nex et al. 2013, Nyaruhuma, et al. 2012a, Nyaruhuma, et al. 2012b).

The most popular way of reliably determining orientation of images is aerial triangulation by bundle block adjustment, unfortunately classical photogrammetric workflow is not suitable for oblique images. Experiments with orientation of this type of data with digital photogrammetric workstation would not provide fully satisfactory results (Jacobsen, 2008; Gerke and Nyaruhuma, 2009). As a consequence the multi-step orientation method was developed and used, these methods assuming separation images into sub-block by looking direction and orientation oblique images directly to adjusted nadir images (Wiedemann and More, 2012) or separate and independent adjustment of each view direction (Hu, et al. 2015).

One of the major reasons why the classical photogrammetric software is not suitable for orientation of oblique images are different orientation angels. Photogrammetric workflow for years was optimized to work with nadir images with similar scale.
Oblique images have very different view angle, tilting of optical axis means that in different parts of image there is a different scale. Traditional algorithms used in photogrammetry in order to extract tie points are not able to deal with this type of image distortion. Furthermore, most of this algorisms are not invariant to affine distortion whose character have differences of view between nadir and oblique images (Xiao et al. 2013).

In recent years interesting studies have been published on the proposed methods for oblique image orientation (Rupnik et al. 2013 Rupnik et al. 2015), using existing algorithms such as SIFT, or proposed new algorithms dedicated to matching oblique images. Searching for ways to improve automatic aerial triangulation for oblique images Yang et al. (2012) proposed a multi-stage algorithm based on SIFT matching. Another solution proposed Xiao et al. (2013) is the using approximated exterior orientation elements in NAIF algorithm (Nicer Affine Invariant Feature),. Another method is proposed by Hu et al. (2015).

Unfortunately, the availability of tools described in the abovementioned studies is still limited and therefore the oblique image orientation still causes trouble in everyday applications. However, in recent years a whole variety of programs successfully used in other segments of market have arisen. They have been developed to some extent regardless of the methods used in large-format (classical) photogrammetry. This software is related to orientation of images from Unmanned Aerial Vehicles (UAV) and Close-Range Photogrammetry. This software based usually on the structure from motion algorithms. Imaging captured in those branches of photogrammetry has often a very diverse geometry - similar to the oblique images. A major problem when using these types of methods to the orientation of oblique aerial images can be computation performance (Karel and Pfeifer, 2015).

The aim of this study was to verify whether it is possible to effectively orientation oblique images in the software used for the

\footnotetext{
* Corresponding author
} 
orientation of images from UAVs and Close-Range Photogrammetry. For this purpose, two quite popular software AgiSoft PhotoScan and Pix4D Mapper Pro were tested in presented research

\section{TEST AREAS}

During experiments three test areas was used, all data was acquired by Polish Photogrammetric Company - MGGP Aero. Cameras which were used are combination of five cameras IGI DigiCam in maltan cross, with tilt angle of 45 degrees. Three of them were $39 \mathrm{Mpx}$ (nadir one has $50 \mathrm{~mm}$ focal length, forward and backward looking cameras have $100 \mathrm{~mm}$ focal length), another two were $50 \mathrm{Mpx}$ (left and right looking also with 100 $\mathrm{mm}$ focal length). All cameras were calibrated and calibration parameters were used as precalibrated values during selfcalibration process. The platform on which cameras are placed is equipped with a GNSS/INS system. The accuracy of the $f$ initial value of the $\mathrm{EO}$ by these systems has been defined a posteriori of $10 \mathrm{~cm}$ for translation and 0.5 degrees for the rotation.

Two of the datasets used in the research were blocks for urban areas. They covered the centers of the two Polish cities of Katowice and Wroclaw. Data for Katowice was acquired in May 2014, and included the 1070 block shots (854 oblique and 216 nadir) with an average GSD of $9 \mathrm{~cm}$ and overlap of $60 \%$ in line and $30 \%$ between the strips. For the test area 56 ground control points were measured in photogrammetric intersection (Fig. 5). The measurement was performed on large-format Images collected with DMCII 230 camera with GSD of $10 \mathrm{~cm}$. As control points selected identifiable manhole covers or rarely road markings were selected.

The flying mission for second test area - Wrocław was carried out in May 2015. 3430 oblique from four cameras were acquired with overlap of $60 \%$ in line and between strips. Avarage GSD was $7 \mathrm{~cm}$ and for the block 12 control points was signalled (painted on the road) and measured using GNSS.

The third of the used blocks included the rural areas, in the northern part of Poland . It consisted of 1108 oblique images on the GSD $10 \mathrm{~cm}$ and characterized by a much smaller overlaps of approx. $30 \%$ both in lines and between strips.

\section{EXPERIMENTS AND ANALYSIS}

Experiments were divided into two groups. Firstly, settings and specific options for individual software are tested in order to determine their influence on orientation process. Next, all other factors which are common for any aerial triangulation process (tie points number per image, GCP distribution, usage of nadir images, overlap between images and number of images in block) were examined.

\subsection{Settings exclusive for one of used software}

The first group of experiments was related to configuration parameters unique to each test programs. Agisoft PhotoScan offers two options of preselection pairs during matching. The first of them (Reference) is based on the approximate EO and the average height of the terrain to find common overlaps of images. The second option (Generic) finds the corresponding images using preliminary matching and pyramid images. Tests have been conducted on the block images from Katowice. Time and effectiveness of these adjustments are presented in Table 1. It can be noticed that the use of options Reference accelerates the process of orientation. Despite a good approximation of exterior orientation that use them in tested software decreases the effectiveness of orientation. Parts of the image blocks remain non-oriented. Therefore after further experiments it was decided not to use the approximate angular exterior orientation of both programs.

\begin{tabular}{|c|c|c|c|c|}
\hline No. & $\begin{array}{c}\text { Key } \\
\text { points }\end{array}$ & $\begin{array}{c}\text { Pair } \\
\text { Preselection }\end{array}$ & Time* & $\begin{array}{c}\text { Oriented } \\
\text { images }\end{array}$ \\
\hline 1 & 90000 & Generic & $245 \mathrm{~min}$ & $1065 / 1070$ \\
\hline 2 & 90000 & Reference & $132 \mathrm{~min}$ & $1046 / 1070$ \\
\hline 3 & 60000 & Generic & $257 \mathrm{~min}$ & $1070 / 1070$ \\
\hline 4 & 60000 & Reference & $190 \mathrm{~min}$ & $1018 / 1070$ \\
\hline
\end{tabular}

Table 1. Time and effectiveness of orientation in Agisoft PhotoScan regarding to Pair Preselection Method. *For 1, 2 and 3, 4 experiments different workstations were used so results in time cannot be directly compared between these pairs.

Pix4D has two configuration options which, without modifying the strategy of matching images may have an impact on the orientation results. The first of them is the use of additional geometry verification of matching and the second is subsequent re-matching after the first calculation of the image orientation.

To verify the effect of these two options on the orientation, a series of experiments with a reduced number of key points (to 10 000) was carried out. Firstly, data from Katowice (Tab. 2) where oriented. The block consisted of both oblique and nadir images. Dense and numerous control points allow to 47 check-points and $9 \mathrm{GCP}$ used in orientation

\begin{tabular}{|c|c|c|c|c|c|c|c|c|}
\hline No. & Dataset & Keypoints & $\begin{array}{c}\text { Matches } \\
(\text { median })\end{array}$ & $\begin{array}{c}\text { Geometry } \\
\text { Verified } \\
\text { Matching }\end{array}$ & Rematch & $\begin{array}{c}\text { Time } \\
{[\mathrm{min}]}\end{array}$ & $\begin{array}{c}\text { Oriented } \\
\text { images }\end{array}$ & $\begin{array}{c}\text { Check Point RMS } \\
\text { X/Y/Z }[\mathrm{m}]\end{array}$ \\
\hline 1 & Katowice & 10000 & 2318 & Yes & Yes & 109 & $1068 / 1070$ & $0.05 / 0.08 / 0.09$ \\
\hline 2 & Katowice & 10000 & 2043 & Yes & No & 86 & $1068 / 1070$ & $0.05 / 0.08 / 0.09$ \\
\hline 3 & Katowice & 10000 & 1955 & No & Yes & 446 & $1026 / 1070$ & $0.31 / 0.12 / 1.25$ \\
\hline 4 & Katowice & 10000 & 1078 & No & No & 333 & $1023 / 1070$ & $0.05 / 0.09 / 0.10$ \\
\hline
\end{tabular}

Table 2. Time and effectiveness of orientation in Pix4D regarding to usage of Geometry Verified Matching and Rematch settings.

\begin{tabular}{|c|c|c|c|c|c|c|c|c|c|}
\hline No. & Dataset & Keypoints & $\begin{array}{c}\text { Matches } \\
\text { (median) }\end{array}$ & GVM & ReM & $\begin{array}{c}\text { Time } \\
{[\mathrm{min}]}\end{array}$ & $\begin{array}{c}\text { Oriented } \\
\text { images }\end{array}$ & $\begin{array}{c}\text { GCP RMS } \\
\text { X/Y/Z }[\mathrm{m}]\end{array}$ & $\begin{array}{c}\text { Check Point RMS } \\
\text { X/Y/Z }[\mathrm{m}]\end{array}$ \\
\hline 1 & Wrocław & 10000 & 1947 & Yes & No & 409 & $3427 / 3430$ & $0.04 / 0.03 / 0.03$ & $0.02 / 0.04 / 0.02$ \\
\hline 2 & Wrocław & 10000 & 2323 & Yes & Yes & 872 & $3427 / 3430$ & $0.04 / 0.04 / 0.04$ & $0.02 / 0.02 / 0.01$ \\
\hline
\end{tabular}

Table 3. Time and effectiveness of orientation in Pix4D regarding to usage of Rematch option. 
The results obtained for the block from Katowice, clearly show that the use of Geometry Verified Matching improves the results which is particularly noticeable in the approach 3 and 4 , where due to the reduction of the number of tie points Pix4D had trouble orienting all images (more than 40 remained not-oriented). This also resulted in the extension of computation time. Differences in effectiveness or accuracy between approach 1 and 2 are not noticed.

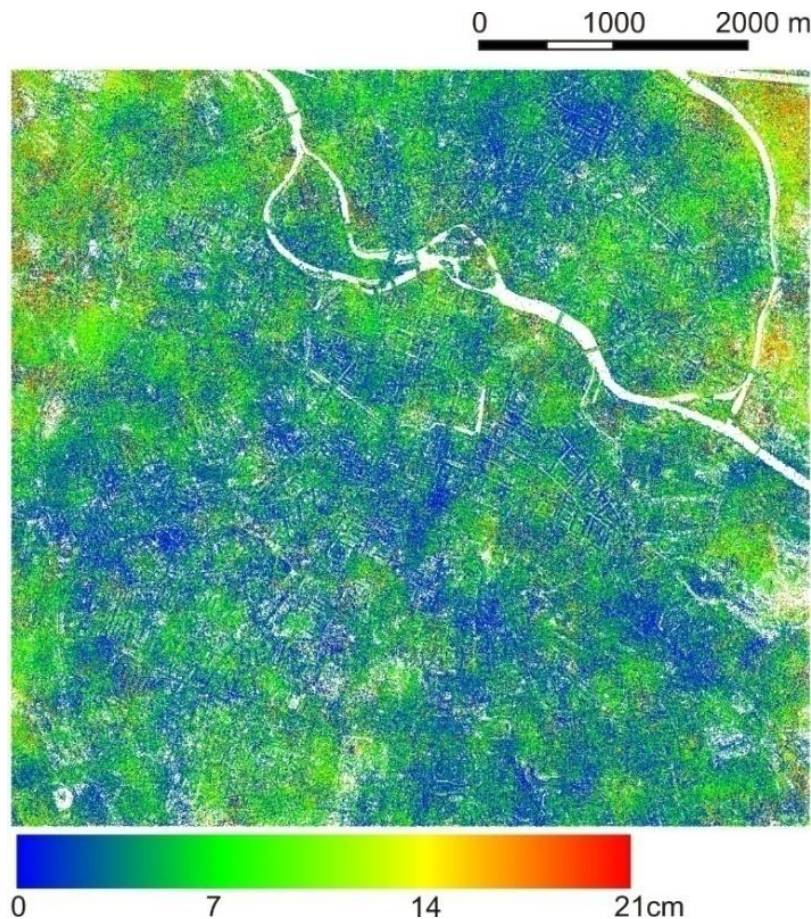

Figure 1. Map of spatial distribution of deviation in tie points height computed with and without Geometry Verified Matching in Pix4D (Wrocław dataset).

Additional experiments were performed for block from Wrocław, which consisted of only the oblique images and had more accurate controls. It could be more likely that the impact of a rematch option would be better seen here. Control was much rarer - during orientation 9 GCPs 4 check points were used. Due to the small number of controls it was decided to further compare the height difference between the tie points extracted in both orientations (Fig. 1). The average height difference was $0.05 \mathrm{~m}$ with a standard deviation (STD) of $0.05 \mathrm{~m}$. In this figure on the distribution minimal systematic differences on the edge of the block can be noticed.

\subsection{Number of tie points}

One of the crucial factors which should have the direct impact on both the time of processing and results of aerial triangulation is number of tie poinst. Decreasing number of tie points should reduce the time of points extraction as well as matching them. However,a reduction of tie point might lead to poor connection between images in block which can have an influence on achieved accuracies. In case of Pix4D the user cannot define desired number of tie points but there is the possibility to define number of key points per image which will be used during matching. Agisoft Photoscan gives both these possibilities.

To evaluate the influence of the reduction of key point numbers a series of aerial triangulation process was carried out. Each process were carriedout fully independently and consists of all steps of processing from key point extraction by image matching to the final adjustment of the block with self-calibration. Dataset from Katowice consist of 1070 images (both oblique and nadir) with 9 GCP and 47 Check Points was used.

Results from Pix4D software clearly show that there is a high potential of increased efficiency in reduction of key point number. Especially because the automatic number of them, which are default settings, is really high (median 86000 per image). The number of key point have almost linear influence on time processing (Fig. 2) and do not influence the achieved accuracy (Fig. 3), It is also visible that the decreasing number of key points below 20000 might influence the effectiveness of orientation (Fig. 4).

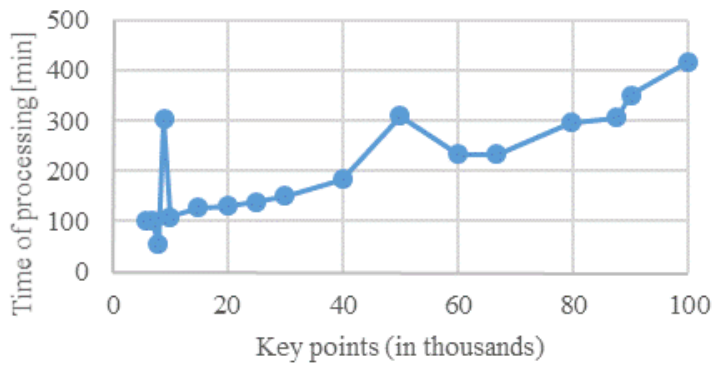

Figure 2. Time of processing (in minutes) in Pix4D of Katowice dataset with different number of key points

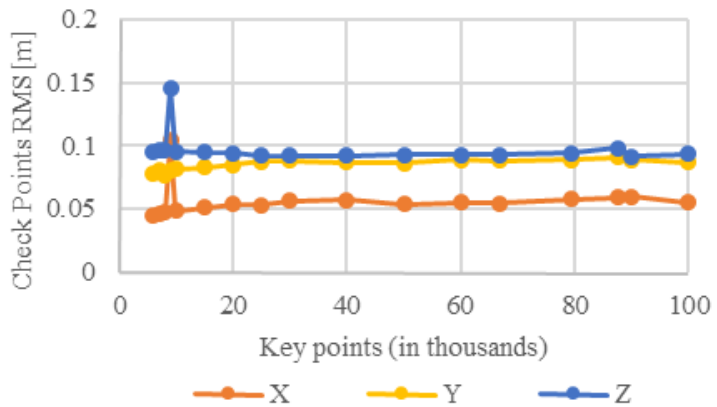

Figure 3. RMS of errors (in meters) achieved on Check Points in Pix4D (Katowice dataset) with different number of key points

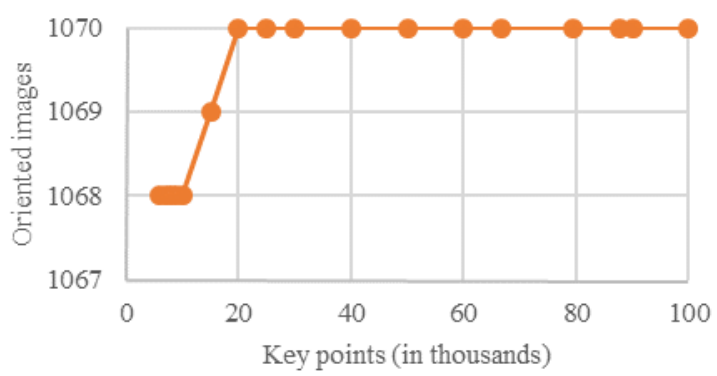

Figure 4. Effectiveness of orientation (Katowice dataset) in Pix4D with different number of key points 
Experiments with reduction of the tie point number in Agisoft Photoscan (Tab. 4) shows that any reduction of tie point number below the default (40000) has direct impact into orientation process stability and effectiveness. There is no visible change in the duration of processing. The only changes involve the number of successfully oriented images.

\begin{tabular}{|c|c|c|c|}
\hline No. & $\begin{array}{c}\text { Key } \\
\text { points }\end{array}$ & $\begin{array}{c}\text { Time } \\
{[\mathrm{min}]}\end{array}$ & $\begin{array}{c}\text { Oriented } \\
\text { images }\end{array}$ \\
\hline 1 & 10000 & 260 & $872 / 1070$ \\
\hline 2 & 15000 & 322 & $865 / 1070$ \\
\hline 3 & 20000 & 260 & $959 / 1070$ \\
\hline 4 & 30000 & 278 & $967 / 1070$ \\
\hline 5 & 40000 & 273 & $1047 / 1070$ \\
\hline
\end{tabular}

Table 4. Time and effectiveness of orientation in AgiSoft

PhotoScan regarding to number of key point per image.

\subsection{GCP distribution}

Another factor that significantly affects the results of aerial triangulation is the number and distribution of ground control points. Due to the large number of tie points and block geometry, it is expected that for a block of oblique images the number of control points can be significantly limited. Reducing the number of GCP is so important that their acquisition is time-consuming, and the number of them can be increased with the division into sub-blocks.

The block from Katowice, due to smaller overlap between the strips can be more sensitive to the reduction of the GCP number Experiments were performed in Pix4D in two variants. The first one used 9 GCP and the second only 4 (Fig. 5). Both variants made two alignments, first using 25000 key points per image and the second of 10000 key points per image. RMS value analysis on Check Points (Tab. 5) showed no significant differences in alignment between using 4 or 9 GCPs. To further verify the impact of ground control points distribution the distance between tie points for both examined variants was created. Such comparison is presented in Fig.5 and this analysis was performed with 10000 key points per image, assuming that the less tie poits you have the impact of analysis should be more visible. The average difference in distance was $4 \mathrm{~cm}$ (with STD of $4 \mathrm{~cm}$ ) and it can be noted that the spatial distribution of the distance between the position of tie points from both alignment does not show any significant systematic component.

\subsection{Adjustment together with nadir images}

The process of relative orientation of images is strongly dependent on their configuration, especially since the occurrence of nadir images is considered. Using them allows it to be much easier to match corresponding points on oblique images with different view direction. However, not for all objectives have oblique images synchronously captured nadir images. In such cases, resignation of nadir images could also improve efficiency.
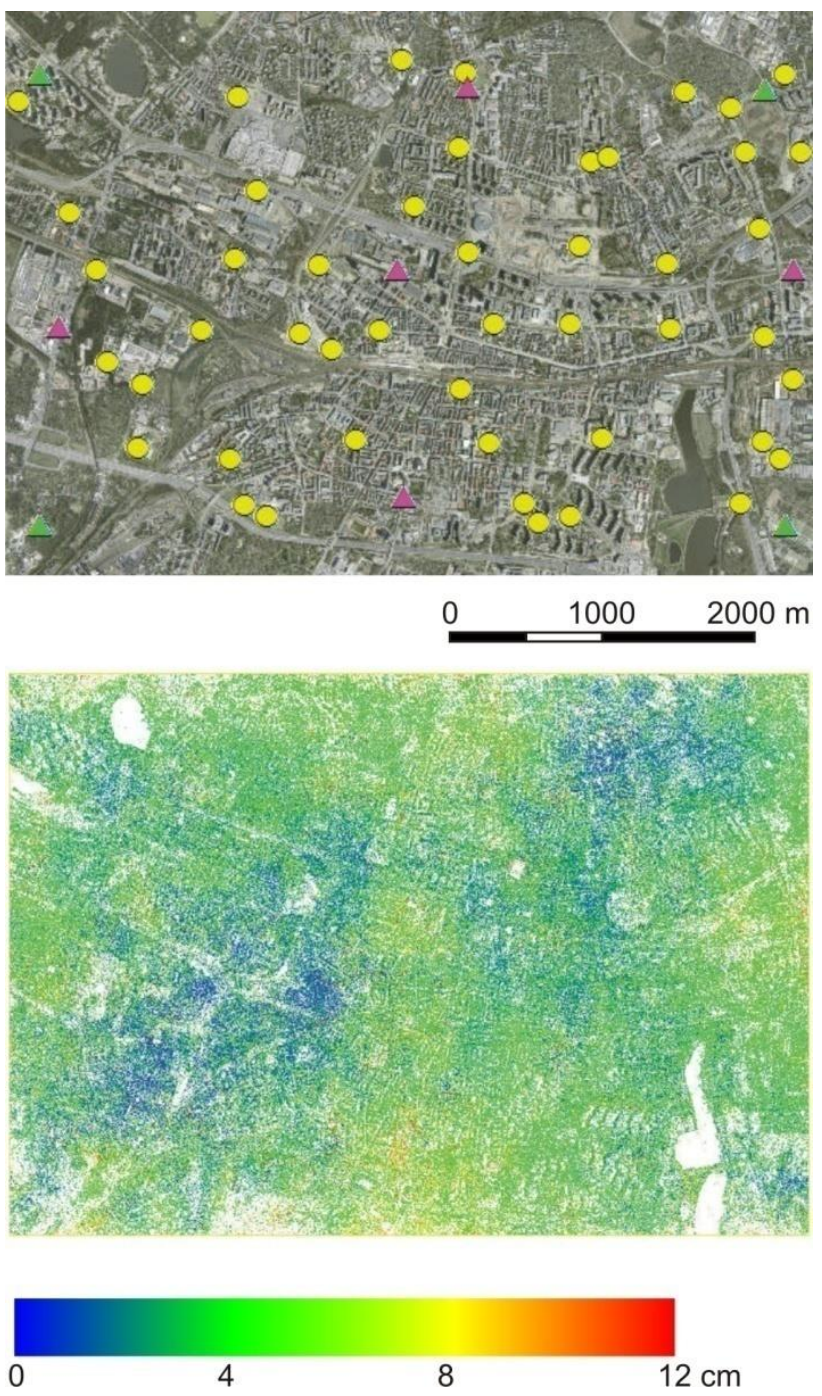

Figure 5. Map of GCP distribution in Katowice dataset. Markers: Yellow - Check points, Green - GCP in both variants, Pink - GCP only in variant with 9 GCPs. Spatial distribution of distances between tie points computed in variant with four and nine GCPs.

\begin{tabular}{|c|c|c|c|c|c|c|c|c|}
\hline No. & Dataset & Keypoints & $\begin{array}{c}\text { Matches } \\
\text { (median) }\end{array}$ & $\begin{array}{c}\text { Oriented } \\
\text { images }\end{array}$ & GCP & $\begin{array}{c}\text { Check } \\
\text { points }\end{array}$ & $\begin{array}{c}\text { RMS GCP } \\
\text { X/Y/Z }[\mathrm{m}]\end{array}$ & $\begin{array}{c}\text { Check Point RMS } \\
\text { X/Y/Z }[\mathrm{m}]\end{array}$ \\
\hline 1 & Katowice & 10000 & 2318 & $1068 / 1070$ & 9 & 47 & $0.06 / 0.06 / 0.05$ & $0.05 / 0.09 / 0.09$ \\
\hline 2 & Katowice & 25000 & 5844 & $1070 / 1070$ & 9 & 47 & $0.06 / 0.07 / 0.05$ & $0.05 / 0.09 / 0.09$ \\
\hline 3 & Katowice & 10000 & 2309 & $1068 / 1070$ & 4 & 47 & $0.05 / 0.04 / 0.05$ & $0.05 / 0.09 / 0.10$ \\
\hline 4 & Katowice & 25000 & 5843 & $1070 / 1070$ & 4 & 47 & $0.04 / 0.05 / 0.06$ & $0.06 / 0.09 / 0.09$ \\
\hline
\end{tabular}

Table 5. The results for GCP number and distribution analysis in Pix4D 


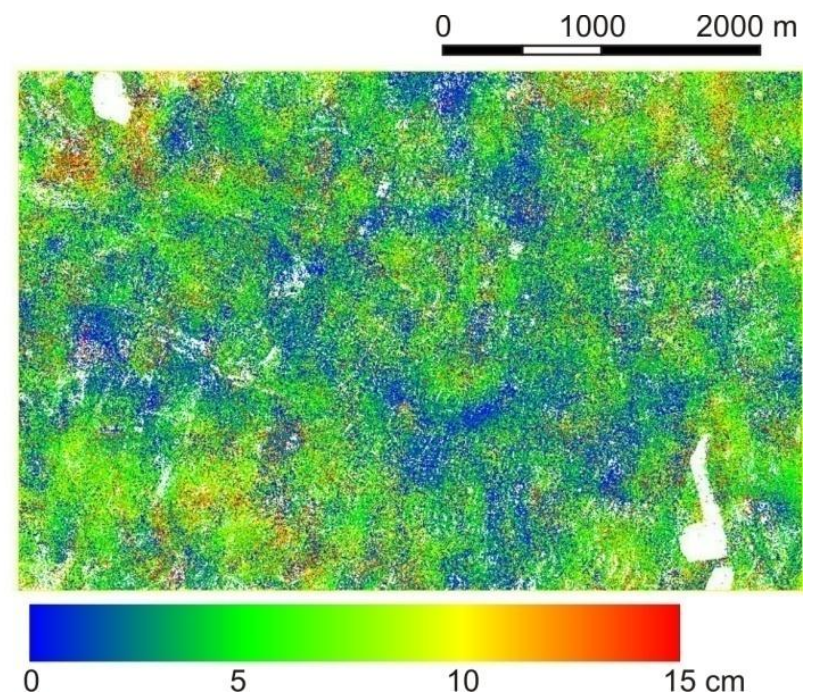

Figure 6. Map of spatial distribution of deviation in tie points height computed with and without nadir images (Katowice dataset).

Fig. 6 presents height differences for tie points extracted in adjustment for blocks where and images were and were not included. As it can be seen using nadir images it is not necessary for an effective orientation, and it does not substantially affect the precision and effectiveness of the orientation.

\subsection{Overlap between images}

A factor that may have a significant impact on the time of matching images is overlaps in a block. More overlaps means that more imagery is considered in adjustment. It should also be noted that tested programs were designed with the aim of processing of images with high overlaps.

A Comparison that was carried out between the time for orientation of the block in Katowice (60/30 overlap) and Wroclaw (60/60) showed no significant increase in computation time omitting an increasing number of images. There are also no differences in the effectiveness of orientation - comparable errors.

Experiments were also carried out on the block with much less overlap of approx. 30/30\%. Attempts at orientation were successful both in Pix4D nor in Agisoft. Major part of block has remained non0oriented or EO parameters were wrong. However, due to other characters of the area, which was a rural area, it is difficult to clearly determine whether the direct cause of such low efficiency of orientation was caused by the lack of full stereoscopic overlap (which is definitely not an advantage) orwhether it was mostly caused by the type of terrain.

\subsection{Number of images - division into sub-blocks}

The last of the examined parameters, which can be crucial for the orientation process, is the number of images used in one alignment. To determine how the increase of the number influences the time of processing in orientation of blocks two datasets (Katowice and Wroclaw) were divided into sub blocks.

For a block from Katowice linear increase was observed for series of adjustments with different number of photos taken to orientation. This caused by small number of in whole block in contrary to a block from Wroclaw where a significant increase in duration time it was observed when large amount of images is considered - 3000 images (Fig. 7) in a single alignment.

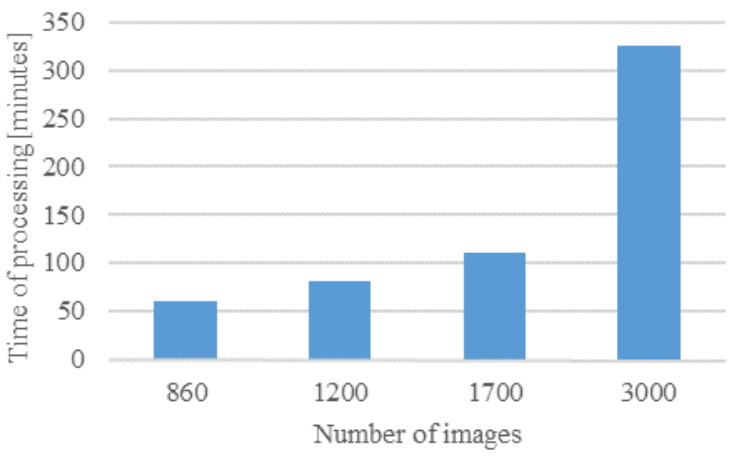

Figure 7. Time of processing (in minutes) in Pix4D depend of number of images (Wroclaw dataset).

It can be expected that the decline in performance orientation depends on how many photos and will also largely depend on the workstation used

\section{SUMMARY AND CONCLUSIONS}

The presented research proves that it is possible to process bundle adjustment of oblique imagery in commercial software. Many variants of adjustment lead to conclusions as follows:

(1) The performed experiments performed using Pix4D and Agisoft have shown that it is possible to use commercial software for effective orientation of the oblique images. However, considering two test programs, Pix4D has a much greater stability. Moreover, in this software orientation of oblique image block can be performed with nadir images simultaneously.

(2) Due to the fact that these programs today cannot effectively use the angular exterior orientation of images, registration with the use of expensive IMU system seems to be pointless. This situation could be changed with the implementation of other algorithms of matching suggested ,for example, by Xiao et al. (2013).

(3) A significant influence on the duration of the aerial triangulation is the number key points which may be significantly reduced in Pix4D with respect to the default settings without having a negative impact on the results.

(4) An important element in the efficient development of large blocks is their division into sub-blocks which improves the productivity of software. In researchan increase of duration was observed when a large number of images was used in aerial triangulation. This can avoid a situation in which it effective alignment will be possible (Karel and Pfeifer, 2015). This solution is much better than with conventional photogrammetry. A block of oblique images is characterized by a more resistant geometry (more tie points on many images, higher overlaps), and can afford a significant reduction in the number GCP without affecting the orientation results.

\section{ACKNOWLEDGEMENTS}

The authors wish to thank MGGP Aero for their friendly attitude and for the data from this company's own projects 


\section{REFERENCES}

Gerke, M. \& Nyaruhuma, P., 2009. Incorporating scene constraints into the triangulation of airborne oblique images. ISPRS - International Archives of the Photogrammetry, Remote Sensing and Spatial Information Sciences, 38(part 1-4-7/W5),

Grenzdorffer, G.J., Guretzki, M. \& Friedlander, I., 2008. Photogrammetric Image Acquisition and Image. The Photogrammetric Record, 23(December), pp.372-386.

Haala, N., Rothermel, M. \& Cavegn, S., 2015. Extracting 3D urban models from oblique aerial images. In 2015 Joint Urban Remote Sensing Event (JURSE). IEEE, pp. 1-4.

Höhle, J., 2008. Photogrammetric measurements in oblique aerial images. Photogrammetrie, Fernerkundung, Geoinformation, 1, pp.7-14.

Hu, H. et al., 2015. Reliable Spatial Relationship Constrained Feature Point Matching of Oblique Aerial Images. Photogrammetric Engineering \& Remote Sensing, 81(1), pp.4958 .

Jacobsen, K., 2008. Geometry of vertical and oblique image combinations. In Remote Sensing for a Changing Europe: Proceedings of the 28th Symposium of the European Association of Remote Sensing Laboratories, Istanbul, Turkey.

Karel W., Pfeifer N., 2015. Analysis of oblique image datasets with OrientAL. In EUROSDR/ISPRS workshop on 'oblique cameras and dense image matching' 19 - 20 October Southampton, UK.

Nex, F., Rupnik, E. \& Remondino, F., 2013. Building footprints extraction from oblique imagery. ISPRS Annals of the Photogrammetry, Remote Sensing and Spatial Information Sciences, II(November), pp.61-66.

Nyaruhuma, P., Gerke, M. \& Vosselman, G., 2012. Verification of 3D Building Models Using Mutual Information in Airborne Oblique Images. ISPRS Annals of the Photogrammetry, Remote Sensing and Spatial Information Sciences, I(September), pp.275280.

Nyaruhuma, A.P. et al., 2012. Verification of 2D building outlines using oblique airborne images. ISPRS Journal of Photogrammetry and Remote Sensing, 71, pp.62-75.

Rau, J., Jhan, J. \& Hsu, Y., 2015. Analysis of Oblique Aerial Images for Land Cover and Point Cloud Classification in an Urban Environment. IEEE Transactions on geoscience and remote sensing, 53(3), pp.1304-1319.

Remondino, F. \& Gerke, M., 2015. Oblique Aerial Imagery - A Review. Photogrammetric Week 2015, pp.75-83.

Rupnik, E., Nex, F. \& Remondino, F., 2013. Automatic orientation of large blocks of oblique images. ISPRS International Archives of the Photogrammetry, Remote Sensing and Spatial Information Sciences, XL(May), pp.21-24.

Rupnik, E. et al., 2015. Aerial multi-camera systems: Accuracy and block triangulation issues. ISPRS Journal of Photogrammetry and Remote Sensing, 101(60), pp.233-246.

Wiedemann, A. \& Moré, J., 2012. Orientation Strategies for Aerial Oblique Images. ISPRS - International Archives of the
Photogrammetry, Remote Sensing and Spatial Information Sciences, XXXIX-B1(September), pp.185-189.

Xiao, X. et al., 2013. Robust and rapid matching of oblique UAV images of urban area. In Proc. SPIE 8919, MIPPR 2013: Pattern Recognition and Computer Vision,. p. 89190Y..

Yang, H., Zhang, S. \& Wang, Y., 2012. Robust and precise registration of oblique images based on scale-invariant feature transformation algorithm. IEEE Geoscience and Remote Sensing Letters, 9(4), pp.783-787. 